\title{
Programas intergeneracionales de actividad física con personas mayores en el ámbito universitario. Un ejemplo práctico para el aprendizaje
}

\author{
Intergenerational programs of physical activity \\ with older people in the university environment. \\ A practical example for learning
}

\author{
Miguel Ángel Morales Cevidanes \\ Universidad Pablo de Olavide \\ mamorcev@admon.upo.es \\ ORCID iD: https://orcid.org/0000-0002-8851-9987 \\ Julio Ángel Herrador Sánchez \\ Universidad Pablo de Olavide \\ jahersan1@upo.es \\ ORCID iD: https://orcid.org//0000-0002-7465-8345
}

\section{Palabras clave \\ - Juego motor \\ - Relaciones intergeneracionales \\ - Conocimiento recíproco \\ - Envejecimiento activo \\ - Adulto mayor}

\section{Resumen}

La mejora de la calidad de vida y las dificultades de la conciliación familiar, son algunas peculiaridades asociadas al S.XXI, que se vinculan al aumento de la longevidad y descenso de natalidad. Cobra especial relevancia el papel de las personas mayores y adolescentes al generar visiones distintas de una misma realidad. Esto hace que ambos grupos tengan un especial protagonismo, por los diversos enfoques que se derivan en un mismo entorno social gracias a sus diferencias intergeneracionales. Nuestra propuesta nace con el objetivo de establecer relaciones en el ámbito académico a través de encuentros de estos grupos generacionales favoreciendo el enriquecimiento mutuo. Por un lado, al mejorar el sentido de la asignatura de Juegos Motores y Habilidades Motrices, en el $1^{\text {er }}$ curso del grado en Ciencias del Deporte, y por otro, al incidir en la mejora de su calidad de vida por la interacción generacional a través de la actividad física, conociendo el efecto positivo del envejecimiento activo.

Con una metodología activa, utilizamos el juego y las dinámicas de grupo como herramienta fundamental. Adecuamos las herramientas de trabajo a las características y conocimiento del grupo, dimos respuesta a sus intereses y motivaciones, con aprendizajes significativos para los estudiantes. Establecimos reciprocidad en el aprendizaje, para que los mayores conocieran los códigos del lenguaje e inquietudes de los adolescentes en un contexto de enseñanza formal. Como principal conclusión obtuvimos una alta valoración de la experiencia y enriquecimiento afectivo social de ambos colectivos. 


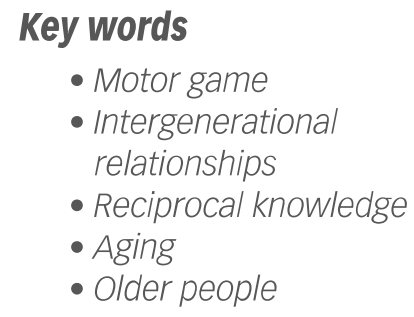

\section{INTRODUCCIÓN}

Año a año, gracias al incremento de la esperanza de vida y con los efectos de la sociedad del bienestar, la concepción del envejecimiento ha ido evolucionando. Partíamos del concepto tradicional de vejez, asociada al cada día más peyorativo término viejo. En él subyace una cierta incapacidad, carencias y fin de los días. Hoy en día avanzamos hacia una concepción más acorde con la realidad, donde envejecer no tiene por qué traer consigo la inminente aparición de limitaciones. A todo ello han contribuido muchos factores, entre ellos las diferentes apuestas políticas y sociales. No podemos obviar el cada vez mayor peso que va teniendo en nuestra sociedad el colectivo de personas mayores. Colectivo que día a día está mejor organizado, bien asociado y mucho más visible. Sobre todo, visibles a una sociedad que no pocos años atrás, ni veía, ni atendía más allá de los entornos familiares.

Así, hoy en día encontramos una visión positiva del envejecimiento, apostando por favorecerlo de forma activa, autónoma y digna. En definitiva, hacer que las personas mayores cada vez sean más partícipes en y de la sociedad, proyectando a ésta una visión positiva de esta etapa vital. En este siglo XXI, se nos plantea un desafío importante al que hay que hacer frente, no es otro que la distancia intergeneracional. Es probable que se haya visto favorecida, como consecuencia del significativo incremento de la esperanza de vida, junto a la convivencia con diversas generaciones en una misma realidad social. Tal es así, que los agentes sociales tendrán como reto el poner en marcha mecanismos que de alguna manera impliquen a todas las generaciones que conviven en un mismo entorno, colaborando entre sí para afrontar con el mayor de los éxitos, tanto las necesidades personales, grupales y comunitarias (Sánchez, Kaplan y Sáez 2010, 28).

En relación con las personas mayores, jugará un papel fundamental en la sociedad actual, la promoción del envejecimiento activo. Éste se está constituyendo como un pilar básico de la misma y para ello es primordial tener en cuenta elementos como la ciudadanía, la importancia del aprendizaje permanente, vivir de forma positiva el propio proceso de envejecimiento y, por supuesto, la importancia que se le asigne a las relaciones intergeneracionales, entre otras. El protagonismo que ha de tener la actividad física va a ser herramienta básica para la prevención y mejora de la salud. Al contrario de lo que podemos 
pensar, deberían ir mostrándose datos alentadores respecto a tasas de práctica deportiva en este perfil poblacional, pero aún persisten tasas muy bajas de actividad física, como se refleja el estudio de (Aragonés et al. 2016, 7) sobre actividad física de la Agencia Española de Protección de la Salud en el Deporte donde los autores indican que tan sólo el $6.05 \%$ de la población entre $65-74$ y el $8.85 \%$ de la población de 55-64, realizaba algún tipo de actividad física. Como podemos ver no es suficiente, haciéndose necesario que las administraciones tanto nacionales como autonómicas continúen apostando por políticas que promuevan el envejecimiento activo y entre ellas por un especial interés los programas relacionados con la actividad física. ¿Y sí, además de no sólo pensar en la mejora de la salud, buscamos favorecer el valor social?, que a través de la práctica deportiva pueden significar las aportaciones que las personas mayores puedan realizar a diferentes generaciones, así como enriquecerse de las vivencias que experimenten con otras edades. Podemos encontrar ese valor añadido en los programas intergeneracionales relacionados con actividad física (Morales-Cevidanes 2015, 149).

Con la propuesta que presentamos, lo que pretendemos es dar un paso más en los programas de actividad física con personas mayores que desde hace más de quince años realizamos en la Universidad Pablo de Olavide. Nuestro entorno educativo ha de ofrecer un conocimiento de todas las realidades en las que los futuros profesionales han de desenvolverse, y por qué no buscar interacciones en su propio ámbito formativo. Por otro lado, que mejor forma de darles un valor y un reconocimiento a las personas mayores que se acercan a la actividad físi$\mathrm{ca}$, que ofreciéndoles la posibilidad de participar en la formación de los futuros profesionales para que conozcan sus peculiaridades e inquietudes. Así mismo, se considera que este tipo de actividades tienen gran potencial para activar a voluntarios mayores (Alloza et al. 2019, 21). Para nosotros supone un reto como docentes, el buscar un contexto de aproximación entre ambas generaciones y que fruto de dicha experiencia, los aprendizajes de nuestros alumnos sean significativos, así como que las aportaciones de estos hacia los mayores les hagan mejorar su adherencia a la práctica deportiva.

Muy recientemente la Consejería de Igualdad, Políticas Sociales y Conciliación (2020) de la Junta de Andalucía, ha presentado el 1 er Plan Estratégico Integral para Personas Mayores en Andalucía 2020-2023, en el que hay una apuesta firme por los programas que promuevan la solidaridad intergeneracional y donde creemos puede tener buena cabida el trabajo que le trasladamos.

\section{LOS PROGRAMAS INTERGENERACIONALES}

No debemos limitarnos a considerar que envejecimiento activo son sólo políticas para personas mayores, deberíamos ir más allá, hemos de considerar que estás políticas se conciben o han de ir encaminadas, a poder llegar a ser mayores con la mayor calidad de vida y en las mejores condiciones, pudiendo mantenerlas durante el mayor tiempo posible. De ello hemos de considerar que son tres los sustentos fundamentales del envejecimiento activo: Salud, Seguridad y Participación. Por otro lado, cabe destacar que Organización Mundial de la Salud (OMS, 2020) ha establecido La Década del Envejecimiento Saludable 2020-2030 como la principal estrategia para lograr y apoyar las acciones destinadas a construir una sociedad para todas las edades, y entre ella cuatro acciones, $1^{\text {a }}$ Cambiar la forma en que pensamos, sentimos y actuamos hacia la edad y el envejecimiento, $2^{\text {a }}$ Asegurar que las comunidades fomenten las capacidades de las personas mayores, $3^{a}$ Ofrecer atención integrada centrada en la persona y servicios de salud primaria que respondan a las personas mayores y por último, $4^{a}$ Brindar acceso a la atención a largo plazo para las personas mayores que la necesitan.

El éxito de estos pilares y estrategia radica entre otros, en una adecuada relación y convivencia con la intergeneracionalidad, invitando a que todos seamos participes del proceso de envejecimiento de una forma activa. Pero no se conseguirían desarrollar en la sociedad actual sin involucrar al conjunto de las administraciones públicas, a las propias personas mayores y por supuesto a los diferentes actores sociales, siendo uno de los principales los Medios de Comunicación Social (Morales-Cevidanes 2017, 131).

Por tanto, podemos resaltar que el concepto envejecimiento activo incluye la intergeneracionalidad como uno de sus principios básicos, entendiendo el concepto según Beltrán y Rivas $(2013,277-294)$, como una propuesta que va más allá de la interacción entre personas de generaciones extremas dentro del ciclo vital-niños y personas mayores-, sino que tiene que dar paso a un cruce de relaciones solidarias entre todas las generaciones, que permita la eliminación de barreras discriminatorias contra las personas por razón de su edad. Una sociedad para todas las edades según la Organización de Naciones Unidas (ONU), es aquella que ajusta sus estructuras y funcionamiento, sus políticas y planes a las necesidades y capacidades de todos, aprovechando por tanto sus posibilidades para beneficio propio, con el objetivo de fortalecer la solidaridad mediante los principios de equidad y reciprocidad entre generaciones (ONU 2002). La Solidaridad Intergeneracional tuvo un punto 
de inflexión en la Asamblea que se celebró en Madrid en 2007. Desde ese momento se concibió como uno de los pilares de la cohesión social, por lo que desde entonces se van a favorecer las iniciativas destinadas a sensibilizar a una sociedad, sobre el potencial tanto de jóvenes como personas mayores, para que se contribuya a dicha solidaridad intergeneracional y al envejecimiento (CEPE 2007 citado en Sánchez, Kaplan y Sáez 2010).

Con esta consideración los programas intergeneracionales han de dar a todos los grupos de edad roles significativos y deben crear, de alguna manera, situaciones y realidades en los que estén implicados. La participación en actividades comunitarias inclusivas viene a contribuir a una mejora de la calidad de vida de las personas. (Flores, Ortega, y Vallejo 2019, 140). Es en ese ámbito donde una institución como la universidad podría plantear propuestas que favorecieran estas iniciativas. Tal fue el alcance desde ese momento que, en abril de 2008, desde el Parlamento Europeo, se convocó una conferencia internacional con el título "Solidaridad intergeneracional por una sociedad cohesionada y sostenible". En el marco de este evento se propuso declarar 2012 como "Año Europeo del Envejecimiento Activo y de la Solidaridad Intergeneracional".

Ante nuestra propuesta deberíamos preguntarnos ¿qué es un programa intergeneracional?, y ver como diferentes autores y organizaciones han definido al mismo. Los programas Intergeneracionales quedan descritos en el National Council on Aging como aquellos que "tienen como objetivo incrementar la cooperación, interacción o el intercambio entre dos generaciones a partir del intercambio de experiencias y conocimiento entre jóvenes y mayores" (Duggar 1993).

Siguiendo con otro organismo internacional como el consorcio internacional para los Programas Intergeneracionales, recogemos la definición de éstos, como "los programas intergeneracionales son medios para el intercambio intencionado y continuado de recursos $y$ aprendizaje entre las generaciones mayores y las más jóvenes con el fin de conseguir beneficios individuales y sociales" (citado en Sánchez y Díaz 2005). Según McCrea, et al, 2004 (citado en Sánchez 2007), "Los programas intergeneracionales son vehículos para el intercambio determinado y continuado de recursos y aprendizaje entre las generaciones más viejas y más jóvenes para lograr beneficios individuales y sociales".

De las diversas concepciones aportadas sobre los programas intergeneracionales, podemos percibir algunas diferencias, pero en todos se repiten elementos definitorios comunes (Sánchez 2007, 16-36), como son la participación de personas distintas generacio- nes, las propuestas de actividades con el objetivo de alcanzar beneficios para todas las personas implicadas y el mantener diferentes tipos de intercambios de forma continua entre todos los participantes.

Queda más que evidenciado que los programas intergeneracionales son un instrumento más de participación social. En ellos se dan cita personas de diferentes edades y gracias a la actividades propuestas, tiene lugar el intercambio de experiencias, de aprendizajes y de relaciones, con el objetivo por un lado de reforzar los beneficios de la propia propuesta, y por otro, generar vivencias positivas tanto a nivel individual como colectivo. En definitiva, se busca entender la sociedad para todas las edades.

La implantación de nuestra propuesta intergeneracional, dentro de la formación de los estudiantes de la Facultad de Ciencias del Deporte de la Universidad Pablo de Olavide, busca continuar con la labor que llevamos a cabo en relación con el envejecimiento activo. Es a través de la promoción de estilos de vida saludable como es el ejercicio físico y el juego, manteniéndolos mentalmente activos y ahora incorporando el trabajo con los más jóvenes. El trabajo está enmarcado en las propuestas del Aula Abierta de Mayores de la Universidad y en consonancia con sus programas intergeneracionales. En ellos se trata de concienciar a la población de todas las edades de lo importante que es para la mejora de su calidad de vida, no solo con relación al ejercicio sino en lo que se refiere a las relaciones sociales.

El sentido de estos programas radica en la generación de algún tipo de vínculo entre los intervinientes, que les ayude a entender el porqué de su forma de proceder, procurando repercusiones positivas sobre ambos, que de alguna manera contribuya a adecuar la formación de los jóvenes, en nuestro caso, y por otro, poder sentirse miembro de un grupo y sentimientos de utilidad en los mayores. El factor relacional que podemos extraer de los programas intergeneracionales es el que en este proyecto más nos interesa.

Según el Libro Blanco del Envejecimiento Activo (Causapié et al. 2011), las relaciones fruto de estás interacciones ayudarán a envejecer mejor cuando:

- Faciliten a personas y comunidades envejecer del modo que les resulte más satisfactorio en sus respectivos contextos.

- Ayuden a aumentar el contacto y la participación social de quienes van tomando conciencia de su envejecimiento.

- Sean cauces para el compromiso y la contribución de personas de cara a conseguir que los entornos de vida sean más apropiados para envejecer mejor. 
- Propicien que todas las personas, con independencia de su edad y sus capacidades, puedan optimizar su calidad de vida y así envejecer mejor.

Por tanto, todo ello repercutirá sobre su estado físico, ya que esa mejora de su autoestima provocará en muchos casos cambios de estado de ánimo, incremento de su vitalidad y una mayor disposición a realizar nuevas y diferentes actividades. La puesta en marcha de actividades intergeneracionales da como beneficio un aumento de la autoestima, una revalorización de la vejez y la creación de una concienciación generacional (Cano et al. 2019, 38).
No sólo saldrán beneficiados de estas propuestas las personas mayores, sino que este tipo de programas intergeneracionales, a los más jóvenes también les aportarán una mejor adecuación de su realidad profesional. También el sentido de utilidad esta presente, al haber contribuido al disfrute a través de sus propuestas, así como de forma indirecta haber adquirido un cierto compromiso o mejor dicho una cierta responsabilidad social, con estas personas mayores.

Del conjunto de beneficios que genera este tipo de programas, a modo recopilatorio podemos destacar de la propuesta de Dupont y Letesson (2010), los siguientes:

Tabla 1. Beneficios logrados tras la participación en un Programa Intergeneracional

\begin{tabular}{|c|c|}
\hline $\begin{array}{c}\text { El encuentro con otras generaciones permite a las } \\
\text { generaciones jóvenes... }\end{array}$ & $\begin{array}{c}\text { El encuentro con otras generaciones } \\
\text { permite a las generaciones mayores... }\end{array}$ \\
\hline $\begin{array}{c}\text { Prepararse para crecer, para envejecer } \\
\text { y también para morir. }\end{array}$ & $\begin{array}{c}\text { Ser productivas y activas; } \\
\text { transmitir lo que han vivido, su historia. }\end{array}$ \\
\hline $\begin{array}{c}\text { Entrar en la historia humana y en su propia historia } \\
\text { personal; construir su identidad personal. }\end{array}$ & $\begin{array}{c}\text { Aprender sobre las nuevas tecnologías, } \\
\text { nuevos conocimientos, familiarizarse } \\
\text { con descubrimientos recientes. }\end{array}$ \\
\hline $\begin{array}{c}\text { Transmitir su realidad, su punto de vista } \\
\text { sobre la vida. }\end{array}$ & Forjar nuevas relaciones interpersonales. \\
\hline $\begin{array}{c}\text { Abrir la mente, estimular el aprendizaje de la vida, } \\
\text { las relaciones humanas. }\end{array}$ & Romper el aislamiento y la soledad. \\
\hline
\end{tabular}

Fuente: extraído de Dupont y Letesson (2010)

\section{LA ACTIVIDAD FÍSICA COMO REFERENCIA DEL PROGRAMA INTERGENERACIONAL}

Como hemos ido refiriendo, lo que queríamos conseguir con nuestra propuesta era dar un paso más en la consideración que, los programas de actividad física con las personas mayores tienen en muchos casos, que no es otra que una mera práctica de actividad física. Por infinidad de autores, está más que demostrado los efectos positivos de los programas de actividad física en personas mayores. (Varo, Martínez y Martínez-González 2003, 668; Pérez Fuentes et al.,2012, 24; Rodríguez-Hernández et al. 2014, 41). Diversos autores nos han trasladado en diferentes ocasiones, que el proceso de envejecimiento es un proceso que se produce en diferentes planos. No sólo hay que considerar el plano físico, sino también el social y el psicológico, por lo que la calidad de vida de los adultos mayores hay que pretenderla en esos tres planos. Así hemos de tener presente que la Organización Mundial de la Salud, incorpora a la salud el concepto de bienestar físico, psíquico y social. En definitiva, los mayores envejecerán de forma más satisfactoria, cuanto más activos se mantengan, tal y como sostiene la teoría de la actividad (Papalia y Wendoks 1997).

La Organización Mundial de la Salud (OMS, 2001), utiliza el término envejecimiento activo en este sentido: "El envejecimiento activo es el proceso por el cual se optimizan las oportunidades de bienestar físico, social y mental durante toda la vida con el objetivo de ampliar la esperanza de vida saludable, la produc- 
tividad y la calidad de vida en la vejez". En definitiva, potenciar al máximo la disponibilidad en la vida cotidiana, con la mayor autonomía posible.

Según Romero, Hita y Martos (2015), en su artículo "la socialización de las personas mayores en el parque biosaludable", recoge varias preocupantes conclusiones. Por un lado, los usuarios más jóvenes, "intrusos" en unas infraestructuras que no se han creado específicamente para ellos, son bienvenidos por los usuarios mayores como fuentes potenciales de una interacción social significativa. Por otro, que la concepción de la actividad física por parte de estos usuarios jóvenes, que usan el parque únicamente para ejercitarse, les conduce a rechazar o desincentivar esa posible interacción social significativa con los usuarios mayores. Y una más, en la que indica que el parque biosaludable ofrece una posibilidad de contrarrestar en cierta medida el proceso de "expulsión" de la vida activa que supone la transición a la vejez. En la medida en que se mantiene la separación intergeneracional, dicha posibilidad sigue sin materializarse, y el parque se convierte en otro escenario donde representar, asumir e interiorizar el rol social de persona anciana.

\section{PROGRAMA INTEGENERACIONAL ACTIVIDAD FÍ- SICA EN LA UNIVERSIDAD PABLO DE OLAVIDE}

Centrándonos en nuestra propuesta, el programa intergeneracional que comenzamos en el curso 2018/19, se concretó como una acción más en el conjunto de intervenciones que realizábamos en el Aula Abierta de Mayores de la Universidad Pablo de Olavide. Ante el ofrecimiento de ésta en participar en nuevas propuestas, decidimos realizarla con un enfoque significativo con relación al aprendizaje de nuestros estudiantes. Así lo organizamos, para que fuesen los estudiantes de $1^{\text {er }}$ curso del Grado en CC. de la Actividad física y el deporte en la asignatura de Habilidades Motrices y Juegos Motores.

Al inicio de curso se expuso a los estudiantes matriculados, que en un determinado periodo del cuatrimestre asistirían a las enseñanzas prácticas un grupo de personas mayores, que participarían de forma conjunta en dichas sesiones y que sus intervenciones habrían de ajustarse a la realidad que en ese momento se encontraran. Por otro lado, asistimos a los municipios de las aulas de mayores que iban a participar en el programa para exponerles lo que se encontrarían a su llegada a la Universidad. Se tuvo presente que, para desarrollar las actividades programadas, se requería que la persona mayor fuese capaz de llevar a cabo sus propios cuidados y que pudiera realizar de forma autónoma actividades de la vida cotidiana.
La idea primordial era que todos se sintieran alumnos/as de las clases que se iban a vivenciar y percibieran que el desarrollo de éstas seguían las mismas pautas que en el proceso de enseñanza-aprendizaje que se estaba llevando en ese momento. A los estudiantes se les ofrecieron conocimientos básicos sobre el envejecimiento, explicándoles lo que supone esta etapa, llena de cambios a muchos niveles, a nivel físico, el organismo ya no funciona de la misma manera, se va ralentizando las tomas de decisiones, la velocidad de reacción, un aumento de la fragilidad y ciertas las limitaciones para llevar a cabo diversas actividades de la vida diaria, etc.. A través de las actividades que se desarrollaron a lo largo del programa se trataron aspectos relativos al estilo de vida, la importancia de mantenerse activo y moverse, pero también la importancia de descansar y en nuestro caso el jugar. Aquí destacamos que, al jugar, en muchos casos se pondrá en disposición aspectos relacionados con la toma de decisiones, comprensión del mensaje, memoria, etc.., obligando de algún modo a las personas a mantenerse activas mentalmente, trabajos de atención, rapidez y precisión, como apuntan algunos autores (Echauri, Pérez y Marín 2002).

También a nivel cognitivo como a nivel psicológico y social, se le aproximó a esta etapa de la vida. La conjunción de intereses entre los aspectos sociales y físicos se muestran como claro ejemplo de las posibilidades que a través de nuestra propuesta podríamos afrontar, y todo ello en consonancia con muchos de los objetivos del Programa Universitario "Aula Abierta de Mayores de la UPO", donde concretamente podemos encontrar estas finalidades:

5. Aprovechar la riqueza cultural de las personas mayores para potenciar su autovaloración y autoestima.

6. Favorecer un envejecimiento activo y satisfactorio.

9. Promover las relaciones intergeneracionales, facilitando así la tolerancia.

El programa se llevó acabo en el segundo cuatrimestre, y se realizaron 4 sesiones, una vez que ya habían sido impartidas la mitad de las previstas para el curso. Así el alumno al iniciar la asignatura podrá ir preparándose y conociendo la dinámica de las clases para que fuese lo más eficaz posible el desarrollo del programa. Entre otros se establecieron los siguientes objetivos generales:

- Crear entornos de aprendizaje compartidos donde se propicien la interacción entre los mayores y los más jóvenes de diferentes localidades. 
- Promover el intercambio de experiencias, conocimientos y habilidades entre personas de diferentes edades, provocando un aprendizaje más significativo en el alumnado.

- Contribuir a una reconstrucción de la realidad social que los jóvenes tienen sobre las posibilidades motrices de las personas mayores, venciendo los prejuicios y estereotipos que tienen sobre ellos.

- Aportar nuevas fórmulas para mejorar y adecuar el aprendizaje del alumnado procurando un beneficio mutuo entre las generaciones participantes en el programa.

- Implicar y motivar a los estudiantes en la participación en el programa.

- Tener un punto de partida en relación con sus conocimientos y competencias respecto al proceso educativo con personas mayores, previas a la experiencia.

- Superar estereotipos y prejuicios de las personas mayores.

- Realizar una evaluación de las expectativas que tienen respecto al programa, previa a su desarroIlo.

Como aspectos metodológicos a resaltar, utilizamos técnicas de lluvia de ideas, para aflorar las consideraciones en relación con las personas mayores, ... qué es una persona mayor para ellos, cuáles son sus características más significativas, etc.... no sólo con relación a la actividad física sino en otros planos, sociales, culturales, comunicativos, etc...

Por parte del profesorado se trabajó el imaginario social sobre mitos y realidades de las personas mayores utilizándose videos, noticias de prensa, páginas web..., en relación con la Actividad Física. El fin principal era utilizar las ideas que se ponían de manifiesto por los alumnos para ir desmintiendo los mitos que sugieran.

Se le planteó al alumnado que trabajaran y reflexionaran sobre cómo les podían facilitar su aprendizaje. Por otro lado, se preparó también la participación de los mayores antes de venir a la Universidad. Se les presentó el programa, cuál era el sentido, fundamentos y el porqué de éste. Cuestiones como, ¿qué conocen de los jóvenes universitarios actuales? ¿y de los de CC. del Deporte?, ¿qué pueden hacer ellos en una clase de universidad?... Tuvimos que dirigir el enfoque de nuestra propuesta al sentido de utilidad que ellos podían tener, así como el que los estudiantes les pudieran aportar. En general se hizo mucho hincapié en hacerles ver a todos, que cada uno tenía que ser consciente de su propia realidad, que los mayores no quieran ser adolescentes, ni los adolescentes mayores, que cada uno se aceptara en la vivencia conjunta como son, con sus virtudes y sus defectos. Finalmente, tras las diferentes sesiones llegamos al final del curso y se hacía necesario conocer si realmente se había conseguido generar un espacio intergeneracional, en el que mayores y estudiantes universitarios, hubieran compartido algo más que un espacio físico y un conjunto de actividades. Incluso que se pudiera haber generado algún tipo de víncuIo, y que hubiese contribuido a reforzar su apuesta por el envejecimiento activo. Así mismo, fue fundamental conocer si se había producido un aprendizaje, por parte del alumnado, más significativo que si no se hubiese llevado a cabo. Todo ello quedó de manifiesto, en la reflexión final conjunta que mantuvimos con cada municipio de los participantes y su grupo de estudiantes, en los cuestionarios de valoración del alumnado y en el informe recibido por cada uno de los municipios participantes.

\section{CONCLUSIONES}

Con el trabajo realizado y los conocimientos aquí expuestos podemos concluir con las siguientes ideas clave:

Se evidencia la necesidad de visualizar los programas de actividad física, no sólo como mejora de las capacidades físicas, sino como herramienta de fomento de las relaciones sociales, y dar un paso más para concebirla como fuente directa de conocimiento y facilitadora de la formación de futuros profesionales de la actividad física.

Las propuestas de intervención en actividad física deberían garantizar un alto grado de empatía por la actividad, por lo que dar un sentido utilitario y relacional, como propone el programa intergeneracional, hace que se genere.

Los estudiantes expresan lo adecuado que ha sido construir su aprendizaje en una realidad concreta, y su repercusión sobre futuras intervenciones didácticas.

Los responsables de los municipios participantes nos trasladan el incremento de la adherencia de los mayores en sus municipios a programas de actividad física. Como muestra, nos refieren que algunos han sido modificados, adecuándolos a los intereses y sobre todo a sus necesidades del alumnado.

Al igual que otros estudios, se ha puesto de manifiesto el gran potencial para activar a las personas mayores que este tipo de actividades tienen. El hecho de participar en actividades inclusivas contribuye de forma clara a la mejora de su calidad de vida, por la influencia de los factores sociales y de reconocimiento. En definitiva, podemos concluir también, que la 
puesta en marcha de actividades intergeneracionales da como beneficio una mejora sustancial en la autoestima de quienes participan, una revalorización de esta etapa del mayor y la creación de una concienciación generacional a toda la sociedad.

Hecho este último que va a verse incentivado por el Primer Plan Estratégico Integral para Personas Mayores en Andalucía 2020-2023, presentado por la administración andaluza y que dará un impulso definitivo a los programas que promuevan la solidaridad intergeneracional.

\section{REFERENCIAS BIBLIOGRÁFICAS}

Aragonés, María Teresa, Fernández, Pablo y Vega de Seoane, Victoria. 2016. Actividad física de prevalencia de patologías en la población española. Madrid: Agencia española de protección de la salud en el deporte. Ministerio de Educación, Cultura y Deporte.

Alloza, Margarita, Garcés, Meritxel, Lázaro, Ana, Quílez, Agustín, Sánchez,Teresa y Muñoz, María José. 2019. "Programa disfruta de la experiencia, voluntariado de mayores y envejecimiento activo en el medio rural-Andorra (Teruel)". Comunitania. Revista Internacional de Trabajo Social y Ciencias Sociales 18: 21-35.

Beltrán, Alicia y Rivas, Adalver. 2013. "Intergeneracionalidad y multigeneralidad en el envejecimiento y la vejez". Tabula Rasa 18: 277-294.

Causapié, Purificación, Balbotín, Antonio, Porras, Manuel, y Mateo, Adela. 2011. Libro blanco del envejecimiento activo. Madrid: IMSERSO.

Consejería de Igualdad, Políticas Sociales y Conciliación. 2020. "Primer Plan Estratégico Integral para Personas Mayores en Andalucía 2020-2023". BOJA No221 - 16 de noviembre de 2020. https://solidaridadintergeneracional.es/files/biblioteca/documentos/ estudio.pdf

Duggar, Margaret. 1993. Intergenerational programs: Weaving hearts and minds. Tallahassee, FL: Florida Council on Aging, Florida State Department of Education.

Dupont, Cecile y Letesson, Mélina. 2010. Comment developer une action nintergénérationnelle? Bruselas: De Boeck.

Echauri, Margarita. Pérez, Ma José y Marín, Pilar. 2002. Envejecer con Salud. Pamplona: Instituto de Salud Pública. Gobierno de Navarra.

Flores, María José, Ortega, María del Carmen y VaIlejo, Silvia. 2019. "Experiencias inclusivas intergeneracionales: ¿un nuevo horizonte para la inclusión de personas mayores y menores?". Miscelánea ComiIlas. Revista de Ciencias Humanas y Sociales, 77(150): 139-152.
Herrador, Julio y Morales-Cevidanes, Miguel Ángel. 2015. Juegos y Dinámicas de grupo para personas mayores. Barcelona: Editorial Onporsport S.L.

Cano, Leticia, Llera, Ana, Córdoba, Celia, Parra, Ana, Peragón, Mónica y Almazán, Agustín. 2019. “Los beneficios de la intergeneracionalidad en las personas mayores de 80 años". Paraninfo Digital Monográficos de Investigación en Salud Paraninfo Digital 13 (30), 1-2. http://ciberindex.com/c/pd/e30064.

Morales-Cevidanes, Miguel Ángel. 2015. "La incidencia del módulo de actividad física para la mejora de la calidad de vida, en los hábitos saludables, del alumnado del aula de mayores de la Universidad Pablo de Olavide". En Longevidad y salud. Innovación en la actividad física. Congreso Internacional de Actividad Física para personas Mayores, editado por CIMA, 141-157. Universidad de Málaga.

Morales-Cevidanes, Miguel Ángel. 2017. "Medios de comunicación y actividad física en personas mayores". En Actividad física para mayores. Investigación, enseñanza y práctica. Congreso Internacional de Actividad Física para personas Mayores, editado por CIMA, 129-152. Universidad de Málaga.

OMS. 2001. "Salud y envejecimiento. Un documento para el debate". Boletín para el envejecimiento, Perfiles y tendencias. IMSERSO. Observatorio de personas Mayores. 4: 15-26.

ONU. 2002. Informe de la Segunda Asamblea Mundial sobre el Envejecimiento. Nueva York: Naciones Unidas.

OMS. 2020. "Atención integrada para las personas mayores (ICOPE): Guía sobre la evaluación y los esquemas de atención centrados en la persona en la atención primaria de salud. Manual". https://iris.paho. org/handle/10665.2/51973

OMS. 2020. "Década del Envejecimiento". https:// www. paho.org/es/decada-envejecimiento-saludable-2020-2030

Papalia, Diane y Wendkos, Sally. 1997. Desarrollo humano. (6a Ed.). Santafé de Bogotá: Mc. Graw-Hill.

Pérez Fuentes, María del Carmen, Gázquez, José, Molero, María y Mercader, Isabel. 2012. "Un estudio de campo sobre el envejecimiento activo en función de la actividad física y ejercicio físico". Revista Iberoamericana de Psicología y Salud, 3 (1): 19- 37.

Rodríguez-Hernández, Mynor, Araya, Felipe, Ureña, Pedro, Wadsworth, Danielle D. y Solano, Luis. 2014. "Aptitud física y su relación con rasgos depresivos en personas adultas mayores que realizan actividad física". Revista en Ciencias del Movimiento Humano y Salud, 11 (1): 35-46.

Romero-Reche, Alejandro, Martos, Pilar e Hita, Cecilia. 2015. "La socialización de las personas mayores en el parque biosaludable". Revista Iberoamericana 
de Ciencias de la Actividad Física y el Deporte, 4 (3): 21-33

Sánchez, Mariano, Kaplan, Matthew y Sáez, Jesús. 2010. Programas Intergeneracionales. Guía introductoria. Colección Manuales y Guías. Serie Personas Mayores. Madrid: Instituto de Mayores y Servicios Sociales.

Sánchez, Mariano y Díaz, Pilar. 2005. Gerontología. actualización, innovación y propuestas. Madrid: Pearson Prentice Hall.

Sánchez, Mariano. 2007. Programas intergenera- cionales. Hacia una sociedad para todas las edades. Barcelona: Fundación "la Caixa".

Schoenmakers, Birggitte, Buntinx, Frank y Delepeleire, Jan. 2010. "Factors determining the impact of care-giving on caregivers of elderly patients with dementia". A systematic literature review. Maturitas, 66 (2): 191-200.

Varo, José Javier, Martínez, Alfredo y Martínez-González, Miguel Ángel. 2003. "Beneficios de la actividad física y riesgos del sedentarismo". Medicina Clínica. (121): 665-672. 